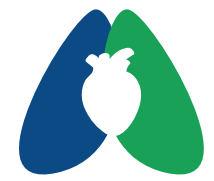

ASSOBRAFIR

CI Ê N C I A

\title{
Análise da capacidade funcional de pacientes cardiopatas na fase I da reabilitação em um hospital público terciário do Distrito Federala
}

\author{
Analysis of functional capacity of cardiac patients in phase I of \\ rehabilitation at a public tertiary hospital in the Federal District
}

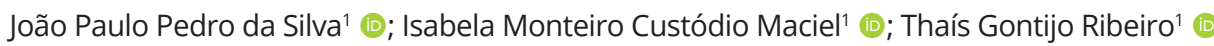

\section{Resumo}

Introdução: As doenças cardiovasculares são responsáveis por mais de 30\% dos óbitos no mundo, sendo um problema de saúde pública que gera limitações na capacidade funcional. Objetivo: Analisar a capacidade funcional de pacientes cardiopatas na Fase I da reabilitação e comparar a função motora na admissão e na alta hospitalar. Métodos: Trata-se de um estudo observacional transversal retrospectivo, cujos dados foram obtidos por prontuários eletrônicos. Foram incluídos prontuários de pacientes de ambos os sexos, maiores de 18 anos, internados na enfermaria cardiológica de um hospital público terciário do Distrito Federal, em maio de 2019 e maio de 2020. Resultados: Foram elegíveis 189 prontuários, houve maior prevalência do sexo masculino $(60,8 \%)$, a média de idade foi de $63,8 \pm 12,74$ anos, tiveram uma média de internação de cerca de 19 $\pm 21,23$ dias e de 13 $\pm 25,13$ sessões de fisioterapia. Entre os fatores de risco não modificáveis o mais frequente foi hipertensão arterial sistêmica $(79,4 \%)$, e entre os modificáveis, o de maior prevalência foi o tabagismo $(15,3 \%)$ seguido de etilismo (13,8\%). O procedimento cirúrgico mais realizado foi o cateterismo (114 dos procedimentos totais). Por meio de análise estatística, o teste de Wilcoxon demonstrou diferença estatística entre a admissão e alta na análise da escala ICU Mobility Scale ( $Z$-3,302 e p=0,001), o que não ocorreu na análise da escala Medical Research Council ( $Z-0,221$ e $p=0,821)$. Conclusão: Ocorreu melhora da mobilidade na alta hospitalar quando comparada à admissão, o que demonstra o impacto da fisioterapia na capacidade funcional destes pacientes cardiopatas.

Palavras-chave: Cardiopatias; Fisioterapia; Reabilitação; Força Muscular.

aApresentação dos dados em evento: resumo expandido apresentado na I Jornada Acadêmica da Fisioterapia do UNICEPLAC.18/05/2021.

${ }^{1}$ Faculdade de Fisioterapia, Centro Universitário do Planalto Central Apparecido dos Santos (UNICEPLAC), Gama, DF, Brasil

Como citar: Silva JPP, Maciel IMC, Ribeiro TG. Análise da capacidade funcional de pacientes cardiopatas na fase I da reabilitação em um hospital público terciário do Distrito Federal. ASSOBRAFIR Ciênc. 2020;11:e42183. https://doi. org/10.47066/2177-9333.AC.2020.0017

Submissão em: Novembro 26, 2020 Aceito em: Maio 24, 2021

Estudo realizado em: Hospital de Base do Distrito Federal, Brasília, DF, Brasil. Aprovação ética: CAAE:

15135219.0.3001.8153 do Instituto de Gestão Estratégica do Distrito Federal e da Escola Superior de Ciências da Saúde, $n^{\circ}$ 3.920.753.

Autor correspondente: Isabela Monteiro Custódio Maciel. E-mail: isabelamonteirop@ gmail.com

\begin{abstract}
Background: Cardiovascular diseases are responsible for more than $30 \%$ of deaths in the world, being a public health problem that generates limitations on functional capacity. Aim: To analyze the functional capacity of cardiopath patients in phase I of rehabilitation and compare motor function at admission and hospital discharge. Methods: This is a retrospective transversal observational study, with data obtained by electronic medical records. Included medical records of both sexes, over 18 years old, hospitalized in the cardiological infirmary of a tertiary public hospital in the Federal District in May 2019 and May 2020. Results: 189 records were eligible, the highest prevalence of males (60.8\%), the mean age was $63.8 \pm 12.74$ years, had an average hospitalization of about $19 \pm 21.23$ days and $13 \pm 25.13$ physiotherapy sessions. Among the most frequent risk factors was systemic arterial hypertension (79.4\%), and among the modifiable, the higher prevalence was smoking (15.3\%) followed by ethylism (13.8\%). The most accomplished surgical procedure was catheterization (114 of total procedures). Through statistical analysis, the Wilcoxon test demonstrated statistical difference between admission and high in the analysis of the ICU Mobility Scale $(Z-3,302 \mathrm{EP}=0.001)$, which did not occur in the analysis of the Medical Research Council $(Z-0,221 \mathrm{EP}=0.821)$. Conclusion: There was improvement of mobility in hospital discharge when compared to admission, which demonstrates the impact of physiotherapy in the functional capacity of these cardiopathic patients.
\end{abstract}

Keywords: Heart diseases; Physiotherapy; Rehabilitation; Muscle strength.

Este é um artigo publicado em acesso aberto (Open Access) e distribuído sob a licença Creative Commons Attribution NonComercial ShareAlike License, que permite uso, distribuição e reprodução em qualquer meio, sem restrições desde que sem fins comerciais e que o trabalho original seja corretamente citado e de forma que não indique endosso ao trabalho feito. A 


\section{INTRODUÇÃO}

As doenças cardiovasculares (DCV) destacam-se como sendo a principal causa de morbimortalidade em todo o mundo, sendo considerado um problema de saúde pública, cerca de 17 milhões de pessoas morrem anualmente por essa enfermidade 1 . No Brasil, mais de $30 \%$ dos óbitos com causa confirmada estão relacionados a DCV².

Existem vários fatores de risco que levam ao aparecimento das DCV, podendo eles serem modificáveis ou não modificáveis. Os fatores de riscos modificáveis incluem níveis séricos elevados das lipoproteínas de baixa densidade (LDL-colesterol), níveis séricos baixos das lipoproteínas de alta densidade (HDLcolesterol), tabagismo, etilismo, hiperglicemia, obesidade, sedentarismo, má alimentação e uso de contraceptivos; e os não modificáveis incluem história familiar de DCV, idade, sexo e raça ${ }^{3}$. Além disso, a qualidade de vida ligada à estes fatores pode influenciar e interferir nas condições de saúde ${ }^{4}$.

A reabilitação cardíaca permite aos cardiopatas retornar o quanto antes à suas atividades de vida diária 5 . Ademais, tem efeitos favoráveis na melhora da capacidade cardiorrespiratória, redução da mortalidade e impacto nas internações, e consequentemente auxilia no aumento da qualidade de vida ${ }^{6}$. Atualmente, os programas de reabilitação são elaborados com a intenção de se obter uma melhora na condição física e na tolerância ao esforço em um curto espaço de tempo ${ }^{7}$.

A reabilitação cardiovascular na fase 1 deve ser iniciada assim que o paciente esteja clinicamente estável e a combinação de exercícios físicos devem ser iniciados de baixa intensidade e aumentarem gradualmente, baseada no esforço do paciente. $O$ seguimento nos programas de atividade física e educação em relação aos fatores de risco são de extrema relevância se forem preparadas ainda na fase hospitalar destes pacientes ${ }^{8}$.

O presente estudo tem o objetivo de analisar a capacidade funcional dos pacientes cardiopatas na fase I da reabilitação, e comparar a funcionalidade na admissão e na alta dos indivíduos que realizaram a fisioterapia.

\section{MÉTODOS}

Trata-se de um estudo observacional longitudinal retrospectivo, após aprovação dos Comitês de Ética em Pesquisa do Instituto de Gestão Estratégica do Distrito Federal (IGESDF) e pela Escola Superior de Ciências da Saúde (ESCS), o qual seguiu respeitando as normas da Resolução n. ${ }^{\circ}$ 466/2012 do Conselho Nacional de Saúde (CAAE: 15135219.0.3001.8153 do Instituto $n^{\circ}$ 3.920.753). $O$ Termo de Consentimento Livre e Esclarecido foi dispensado no estudo pelo uso exclusivo de dados coletados de prontuário e pela impossibilidade de encontrar todos os pacientes em tempo hábil.
A amostra foi composta por análise dos prontuários de pacientes internados na enfermaria de serviço de cardiologia de um hospital público terciário, no período de maio de 2019 e de maio de 2020. Foram incluídos neste estudo prontuários de pacientes de ambos os sexos, acima de 18 anos de idade, internados com alguma cardiopatia diagnosticada na enfermaria do hospital. Foram excluídos os prontuários de pacientes que apresentaram dados insuficientes como as escalas analisadas incompletas.

Para a análise da capacidade funcional foram consideradas as escalas Medical Research Council (MRC) e a ICU Mobility Scale (IMS) na admissão e alta hospitalar, ou o mais próximo da alta e da admissão. Estas escalas avaliam a força muscular e a mobilidade respectivamente, questões relevantes a serem consideradas ao avaliar a capacidade funcional do paciente cardiopata, favorecendo a compreensão do quadro do paciente.

\section{Medical Research Council (MRC)}

A escala MRC avalia a força muscular global do indivíduo de 6 grupos musculares principais, sendo 3 grupos musculares dos membros superiores e 3 dos membros inferiores, são eles: abdução de ombro, flexão de cotovelo, extensão de punho, flexão de quadril, extensão de joelho e dorsiflexão de tornozelo. Cada grupo muscular é avaliado por uma pontuação que varia entre 0 e 5, onde 0 é a ausência de contração muscular e 5 é força muscular normal. O somatório de todos os grupos musculares tem a pontuação máxima de 60 , quanto maior a pontuação, menor a alteração na capacidade funcional ${ }^{9}$.

\section{ICU Mobility Scale (IMS)}

A escala IMS é utilizada para avaliar a capacidade funcional pela mobilidade do indivíduo e pode ser utilizada em pacientes hospitalizados. A escala varia de 0 a 10, uma escala gradual, onde 0 (zero) equivale a ausência de mobilidade, ou seja, não ocorre a movimentação e a pontuação 10 (dez) equivale a uma mobilidade sem alterações, ou seja, ocorre a deambulação independente ${ }^{10}$.

\section{ANÁLISE ESTATÍSTICA}

Todas as análises estatísticas foram realizadas pelo programa SPSS, versão 2,3. Iniciou-se a análise da normalidade da amostra por meio do teste ShapiroWilk, observou-se todas as variáveis não normais. Posteriormente foram analisadas as médias, desvio padrão, porcentagens e variação da caracterização da amostra pelas variáveis: sexo, idade, tempo de internação, as comorbidades e as variáveis relacionadas à fisioterapia. O teste de Wilcoxon foi utilizado para a análise das medianas das escalas MRC e IMS na admissão e na alta. Para todos os testes adotou-se um nível de significância de 0,05 . 


\section{RESULTADOS}

Foram analisados 228 prontuários, dos quais 39 foram excluídos, 19 por não foram localizados no sistema de prontuário eletrônico, 19 por não possuírem evolução da fisioterapia e 1 não apresentava diagnóstico cardiológico. A amostra foi constituída em sua maioria por homens $(60,8 \%)$ e uma média de idade de $63,89 \pm 12,74$ anos (Tabela 1).

Dentre os fatores de risco não modificáveis o mais prevalente foi a hipertensão arterial sistêmica (HAS) presente em $79,4 \%$ dos pacientes, seguido de dislipidemia (45\%) e entre os fatores de risco modificáveis, o tabagismo foi prevalente em $15,3 \%$ dos casos, seguido de etilismo $(13,8 \%)$, sendo a maioria do sexo masculino conforme mostra a Tabela 2.

Em relação às comorbidades, $51,9 \%$ foram consideradas "outras doenças" incluindo câncer, doença de chagas, depressão e AVC (acidente vascular cerebral), seguido de infarto agudo do miocárdio (IAM) prévio $(42,9 \%)$, doença renal $(16,4 \%)$, doença pulmonar $(13,8 \%)$ e doença reumatológica $(5,3 \%)$.

Durante o período de internação (média de 19 21,23 dias) na enfermaria foram realizadas uma média de $13 \pm 25,13$ sessões de fisioterapia, de até 2 sessões por dia, sendo preconizada a saída do leito de forma gradual de acordo com a tolerância do paciente, porém visando sempre o condicionamento cardiopulmonar.

Em relação às condutas fisioterapêuticas, a necessidade de suplementação de oxigênio esteve presente em 34 pacientes. Necessitaram de assistência ventilatória 5,8\% $(n=11)$ dos pacientes, sendo que $3,2 \%(n=6)$ fizeram uso

Tabela 1. Caracterização da amostra estudada ( $\mathrm{N}=189)$.

\begin{tabular}{cc}
\hline Variável & Frequência \\
\hline Idade (média) anos, desvio padrão & $63,89( \pm 12,74)$ \\
Sexo, n masculino $(\%)$ & $115(60,8)$ \\
Dias de internação (desvio padrão) & $12( \pm 21,23)$ \\
\hline
\end{tabular}

\% (porcentagem); n (amostra).

Tabela 2. Fatores de risco modificáveis e não modificáveis associados à condição cardíaca em ordem de maior prevalência para menor prevalência. ( $\mathrm{N}=189)$.

\begin{tabular}{cc}
\hline Variável & $\begin{array}{c}\text { Frequência total }(\%), \\
(\% \text { masculino })\end{array}$ \\
\hline HAS & $\mathbf{7 9 , 4}(\mathbf{6 0}, \mathbf{7})$ \\
DLP & $45(57,6)$ \\
DM & $35,4(55,2)$ \\
Tabagismo & $\mathbf{1 5 , 3 ( 6 5 , 5 )}$ \\
Etilismo & $13,8(84,6)$ \\
\hline
\end{tabular}

HAS (hipertensão artéria sistêmica); DM (Diabetes Melittus); (DLP) dislipidemia. exclusivo de ventilação mecânica invasiva (Tabela 3). Vale ressaltar ainda que, 5,3\% desses pacientes obtiveram alta ainda sob o uso da oxigenoterapia.

Quanto aos procedimentos realizados pelos pacientes do estudo, o cateterismo foi realizado em 114 pacientes e apenas 29 realizaram cirurgia de revascularização do miocárdio, sendo que houve outros procedimentos destacados na Figura 1. A principal justificativa para a maior quantidade de procedimentos considerados menos complexos na área da cardiologia se deve a menor gravidade dos pacientes estudados.

$\mathrm{Na}$ análise de comparação entre os valores das escalas que avaliam a força muscular e mobilidade, observou-se que: a mediana do valor de MRC não apresentou diferença estatística significativa quando comparada a MRC da admissão e da alta (Z -0,221 e p=0,821), e a IMS foi maior na alta quando comparada a admissão $(Z-3,302$ e p=0,001) e teve diferença estatística significativa (Figura 2). Apesar dos valores de MRC serem os mesmos como mostra a Figura 2, este fato ocorreu devido a análise por meio de mediana dos valores.

Pode-se observar pelos dados da amostra que a força muscular da maioria dos indivíduos não estava com alteração significativa na admissão $(M R C=48)$, já a resistência cardiopulmonar que tem relação direta com

Tabela 3. Caracterização da amostra em relação a fisioterapia durante a internação. ( $\mathrm{N}=189)$.

\begin{tabular}{cc}
\hline Variável & $\begin{array}{c}\text { Frequência (média, } \\
\text { desvio padrão) }\end{array}$ \\
\hline $\begin{array}{c}\text { Sessões de fisioterapia (média, } \\
\text { desvio padrão }\end{array}$ & $13,24( \pm 25,13)$ \\
$\begin{array}{c}\text { Oxigenoterapia (frequência, desvio } \\
\text { padrão) }\end{array}$ & $34( \pm 3,8)$ \\
VMNI (frequência, desvio padrão) & $5( \pm 2,6)$ \\
VM (frequência, \%) & $6( \pm 3,2)$
\end{tabular}

\% (porcentagem); VMNI (ventilação mecânica não invasiva); VM (ventilação mecânica).

Principais procedimentos cardiológicos realizados durante a internação $(\mathrm{N}=189)$

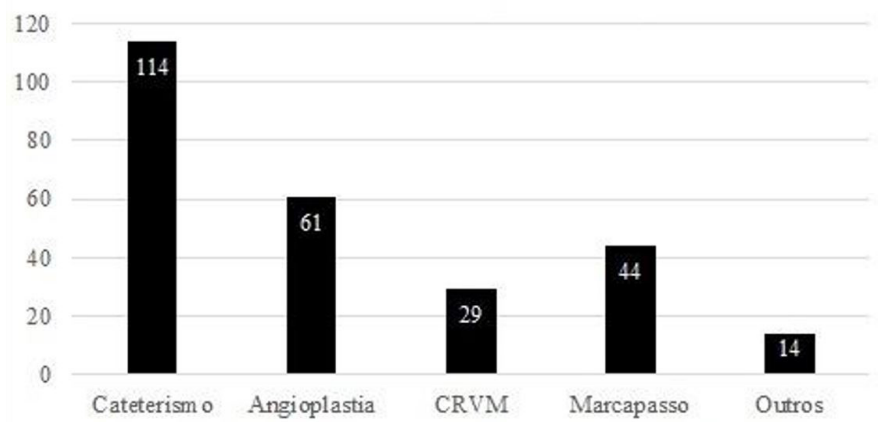

Figura 1. Principais procedimentos cardiológicos realizados durante a internação da amostra estudada.

Legenda: CRVM (cirurgia de revascularização do miocárdio). 


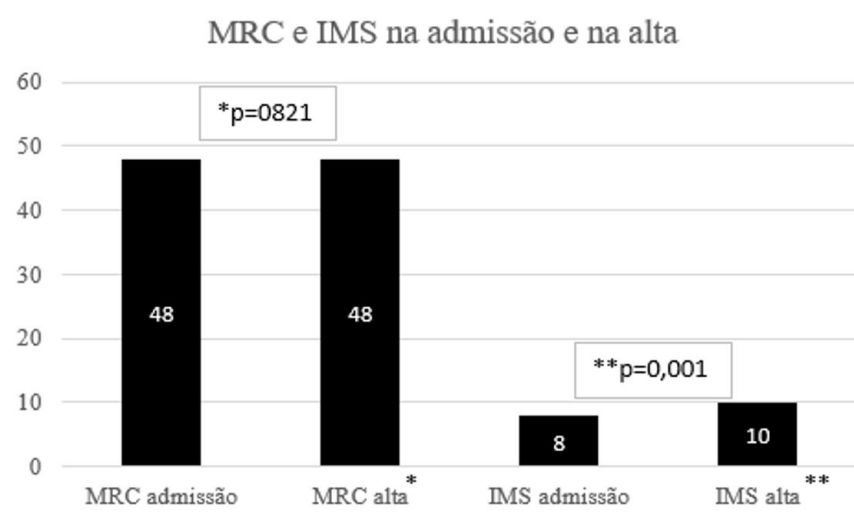

Figura 2. Valores das medianas encontradas entre a admissão e alta das escalas MRC e IMS.

Legenda: MRC (Medical Research Council), IMS (ICU Mobility Scale), considerando $\mathrm{p}=0,005$.

\section{Desfechos da internação}

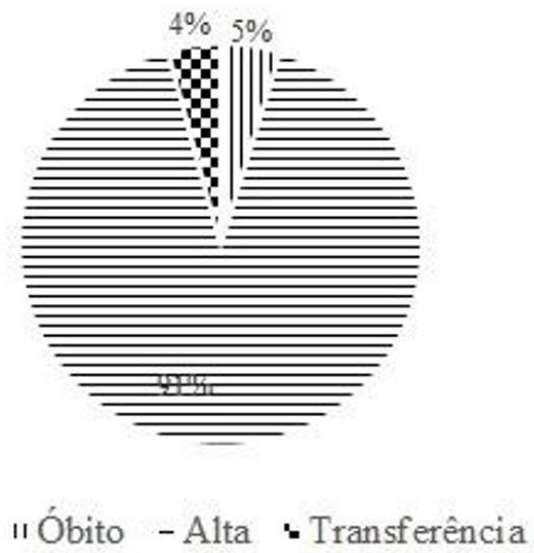

Figura 3. Desfechos encontrados entre os participantes do estudo.

a mobilidade, esteve mais reduzida na admissão (IMS=8). Dentre os principais papéis da fisioterapia, a reabilitação cardiopulmonar tem impacto diretamente relacionado à capacidade pulmonar, resistência e retorno precoce às atividades.

Atentando-se aos dados, que demonstraram a possibilidade dos pacientes da amostra serem menos graves, por causa dos procedimentos considerados mais simples como o cateterismo seguido de angioplastia, a Figura 3 demonstra concordância com esta informação, já que $90 \%$ dos pacientes tiveram alta e apenas $5 \%$ evoluíram à óbito. Observa-se a partir da Figura 3, com a maioria dos pacientes com alta hospitalar, a importância de orientálos a continuar com a reabilitação cardiopulmonar em domicílio para se evitar reinternação hospitalar.

\section{DISCUSSÃO}

A idade dos pacientes deste estudo foi semelhante à outros estudos, como o de Muela et al.(2011), que em uma coorte avaliou os benefícios da reabilitação cardíaca em 88 pacientes cardiopatas, sendo $52,8 \%$ do sexo masculino ${ }^{11,12}$.

O tempo de internação hospitalar pode ser considerado um dos indicativos da complexidade dos casos destes pacientes, já que geralmente casos menos graves permanecem menos tempo internados. Assim como os procedimentos realizados durante a internação, considerados menos complexos na área da cardiologia, no caso do cateterismo e angioplastia, além da elevada taxa de altas hospitalares, se deve à menor gravidade dos pacientes estudados.

Concordando com a média de 18,89 dias de internação apresentada neste estudo, Silva et al. (2014) em um estudo observacional, avaliou a funcionalidade respiratória de 19 pacientes com tempo prolongado de internação hospitalar, sendo $68 \%$ do sexo masculino com média de idade de 65 anos, além disso, concluiu que houve redução da força muscular inspiratória causando prejuízo na ventilação pulmonar ${ }^{13}$.

Apesar de que o tabagismo esteve presente em 15,3\% da população estudada nesta pesquisa, sabe-se que esse é um dos principais fatores de risco modificável que contribui para a ocorrência de doenças cardiovasculares ${ }^{14}$. Outro estudo transversal realizado na PUC de São Paulo avaliou 100 pacientes submetidos à cirurgia cardíaca e mostrou que houve predomínio do sexo masculino (56\%), com idade entre 50 e 70 anos, tendo a HAS, DM e DLP como principais fatores de risco associados ${ }^{15}$.

Um estudo que identificou o perfil dos pacientes submetidos ao cateterismo cardíaco mostrou que extabagistas $(39,2 \%)$ e tabagistas $(18,2 \%)$ também como principais fatores de risco modificáveis ${ }^{16}$. 0 estudo de coorte de Wajner et al. (2017) identificou as causas de mortalidade intra-hospitalar de pacientes com IC, mostrando as comorbidades mais prevalentes no cardiopata: o IAM prévio (infarto agudo do miocárdio), a DPOC (doença pulmonar obstrutiva crônica) e a doença renal ${ }^{17}$.

A reabilitação cardíaca além de auxiliar a controlar os fatores de riscos, ainda reduz a probabilidade de ocorrer complicações secundárias em pacientes com HAS, contribuindo efetivamente para os ganhos fisiológicos decorrentes da atividade física ${ }^{18,19}$. Vargas et al. (2016) em uma revisão da literatura, identificou os principais desfechos clínicos resultantes de programas de reabilitação cardíaca em fase I e ll entre os anos de 2009 e 2014, concluindo que a fisioterapia tem um papel fundamental na reabilitação cardíaca, produzindo alterações autonômicas e no perfil bioquímico dos indivíduos, melhorando a eficiência do sistema cardiorrespiratório, a capacidade funcional e a qualidade de vida ${ }^{20}$.

Nos cardiopatas a oxigenoterapia objetiva corrigir a hipoxemia, e consequentemente, melhorar a oxigenação tecidual, retardar ou amenizar quadros de dispneia e diminuir a carga de trabalho cardiopulmonar. Em um estudo transversal que avaliou as adequações dos dispositivos de oxigenoterapia e identificou os cardiopatas 
e portadores de doenças crônicas como principais indicadores para uso de oxigênio ${ }^{21}$. Como destaque nesse estudo, 10 pacientes $(5,3 \%)$ receberam alta hospitalar ainda sob o uso de oxigênio. Além disso, o estudo exploratório-descritivo de Watanabe et al. (2015) mostrou que há benefícios na capacidade pulmonar dos pacientes crônicos que fazem o uso da oxigenoterapia domiciliar ${ }^{22}$.

A Ventilação não invasiva (VNI) é uma importante ferramenta utilizada no tratamento de pacientes com Insuficiência Cardíaca (IC), quando utilizada de maneira apropriada, proporciona maior conforto respiratório, bem como reduz a necessidade de intubação traqueal e morbimortalidade ${ }^{23}$. Em uma revisão sistemática com meta-análise, Bittencourt et al. (2017) observaram as evidências científicas publicadas até o ano de 2015 sobre o impacto da VNI na capacidade funcional (CF) de pacientes com IC. Os resultados indicaram uma melhora significativa na tolerância ao exercício nos pacientes com IC após intervenção com VNI ${ }^{24}$.

Os estudos de Soares et al. (2016) e Dordetto et al. (2011) analisaram as principais complicações no pósoperatório de cirurgia cardíaca e as características dos pacientes submetidos à cirurgia cardíaca em 2011 e 2016 respectivamente mostrando que a CRVM (58\%) foi o principal procedimento cardiológico realizado seguido de troca valvar (18\%) e valvopatias (14\%) ${ }^{15,25}$.

Quanto aos desfechos, um coorte publicado em 2017 pela Sociedade Brasileira de Cardiologia, estudou o índice de mortalidade intra-hospitalar de 2056 pacientes, desses, $17,6 \%$ evoluíram à óbito durante a internação e 47,4\% por descompensação da insuficiência cardíaca aguda ${ }^{17}$. Já o estudo de Brant et al.(2017) avaliou os diferenciais e variações da mortalidade por doenças cardiovasculares no Brasil em 1990 e 2015, mostrou que houve redução na taxa de mortalidade padronizada por idade de 429 no ano de 1990 para 256 no ano de 2015 a cada 100 mil habitantes e que a taxa nos homens é maior do que nas mulheres ${ }^{26,27}$.

\section{CONCLUSÃO}

A reabilitação na fase I de pacientes cardiopatas, considerados menos graves neste estudo, resultou na melhora da mobilidade no momento da alta hospitalar quando comparada à admissão. Sabe-se que a validade externa deste estudo torna-se fragilizada pelo número amostral, porém existe uma relevância clínica ao demonstrar o impacto da atuação da atuação da fisioterapia na mobilidade de pacientes cardiopatas.

\section{FONTE DE FINANCIAMENTO}

Nada a declarar.

\section{CONFLITO DE INTERESSES}

Nada a declarar.

\section{REFERÊNCIAS}

1. Braig S, Peter R, Nagel G, Hermann S, Rohrmann S, Linseisen J. The impact of social status inconsistency on cardiovascular risk factors, myocardial infarction and stroke in the EPICHeidelberg cohort. BMC Public Health. 2011;11:104. http:// dx.doi.org/10.1186/1471-2458-11-104. PMid:21324154.

2. Schmidt MI, Duncan BB, Azevedo e Silva G, Menezes AM, Monteiro CA, Barreto SM, et al. Chronic non-communicable diseases in Brazil: burden and current challenges. Lancet. 2011;377(9781):1949-61. http://dx.doi.org/10.1016/S01406736(11)60135-9. PMid:21561658.

3. Nascimento BR, Brant LCC, Oliveira GMM, Malachias MVB, Reis GMA, Teixeira RA, et al. Cardiovascular disease epidemiology in Portuguese-speaking countries: data from the global burden of disease, 1990 to 2016. Arq Bras Cardiol. 2018;110(6):500-11. http://dx.doi.org/10.5935/ abc.20180098. PMid:30226906.

4. Barbosa RMSP. Resenha do livro "Atividade Física, Saúde e Qualidade de Vida: conceitos e sugestões para um estilo de vida ativo", de Markus Vinicius Nahas. Rev Bras Ciênc Esporte. 2012;34(2):513-8. http://dx.doi.org/10.1590/S010132892012000200018.

5. Moraes RS, Rodrigues R, De Castro T, Stein R, Serra SM, De Carvalho T. Diretriz de reabilitação cardíaca. Arq Bras Cardiol. 2005;84:431-40.

6. Martin BJ, Arena R, Haykowsky M, Hauer T, Austford LD, Knudtson $\mathrm{M}$, et al. Cardiovascular fitness and mortality after contemporary cardiac rehabilitation. Mayo Clin Proc. 2013;88(5):455-63. http://dx.doi.org/10.1016/j. mayocp.2013.02.013. PMid:23639499.

7. Karaszewski D. Comparison of two models of hospital rehabilitation in patients after coronary artery bypass grafting. Kardiochir Torakochirurgia Pol. 2014;11(1):86-9. http://dx.doi.org/10.5114/kitp.2014.41940. PMid:26336403.

8. Carvalho T, Cortez AA, Ferraz A, Nóbrega ACL, Brunetto AF, Herdy $\mathrm{AH}$, et al. Reabilitação cardiopulmonar e metabólica: aspectos práticos e responsabilidades. Rev Bras Med Esporte. 2005;11(6):313-8. http://dx.doi.org/10.1590/S151786922005000600002.

9. Ferreira NA, Lopes AJ, Ferreira AS, Ntoumenopoulos G, Dias J, Guimaraes FS. Determination of functional prognosis in hospitalized patients following an intensive care admission. World J Crit Care Med. 2016;5(4):219-27. http://dx.doi. org/10.5492/wjccm.v5.i4.219. PMid:27896146.

10. Kawaguchi YM, Nawa RK, Figueiredo TB, Martins L, PiresNeto RC. Perme intensive care unit mobility score and ICU mobility scale: translation into Portuguese and cross-cultural adaptation for use in Brazil. J Bras Pneumol. 2016;42(6):42934. http://dx.doi.org/10.1590/s1806-37562015000000301. PMid:28117473.

11. Klahr PDS, Soares Rocha Oliveira KF, Coronel CC. Perfil epidemiológico e clínico de pacientes encaminhados para Reabilitação Cardiopulmonar e Metabólica fase II. Rev FisiSenectus. 2019;6(2):26-37. http://dx.doi.org/10.22298/ rfs.v6i2.4541.

12. Muela S, Cotchi H, Bassan R, Serra SM. Benefícios da reabilitação cardíaca. Rev Bras Cardiol. 2011;24(4):241-50.

13. Silva BCA, Amorim D, Salício VAMM, Salício MA, ShimoyaBittencourt W. Avaliação da funcionalidade respiratória em pacientes com tempo prolongado de internação hospitalar. J Heal Sci Inst. 2014;32(4):433-8.

14. Barbosa AA, Lima Aires Gomes GP, Roieski IM, Ribeiro Rodrigues ES, Silva Carlotto Herrera SD. Prevalence of cardiovascular risk factors among hypertensive military police 
officers. Rev Enferm UFPE line. 2011;5(10):2374-82. http:// dx.doi.org/10.5205/reuol.2133-15571-1-LE.0510201106.

15. Dordetto PR, Pinto GC, Rosa TCSC. Pacientes submetidos à cirurgia cardíaca: caracterização sociodemográfica, perfil clínico-epidemiológico e complicações. Rev da Fac Ciências Médicas Sorocaba. 2016;18(3):144-9. http://dx.doi. org/10.5327/Z1984-4840201625868.

16. Sousa SM, Bernardino E, Vicelli RMM, Kalinowski CE. Perfil de pacientes submetidos ao cateterismo cardíaco: subsídio para prevenção de fatores de risco cardiovascular. Cogitare Enferm. 2014;19(2):304-8.

17. Wajner A, Zuchinali P, Olsen V, Polanczyk CA, Rohde LE. Causes and Predictors of In-Hospital Mortality in Patients Admitted with or for Heart Failure at a Tertiary Hospital in Brazil. Arq Bras Cardiol. 2017 Out;109(4):321-30. PMid:28977049.

18. Regenga M M. Fisioterapia em cardiologia - da unidade de terapia intensiva à reabilitação. São Paulo: Roca; 2000. 292 p.

19. Mochel EG, Andrade CF de, Almeida DS de, Tobias AF, Cabral R, Cossetti RD. Avaliação do tratamento e controle da hipertensão arterial sistêmica em pacientes da rede pública em São Luis (MA). Rev baiana saúde pública. 2007;31(1);90101.

20. Vargas MHM, Vieira R, Balbueno RC. Atuação Da Fisioterapia Na Reabilitação Cardíaca Durante As Fases I E li - Uma Revisão Da Literatura. Rev Context Saúde. 2016;16(30):85. http://dx.doi.org/10.21527/2176-7114.2016.30.85-91.

21. De Souza K, Córdova PA, De Carvalho JC. Adequações dos dispositivos de oxigenoterapia em enfermaria hospitalar avaliadas por oximetria de pulso e gasometria arterial Resumo A oxigenoterapia consiste na administração de oxigênio acima da concentração ambiental. ASSOBRAFIR Ciência. 2014;5(1):53-64.

22. Watanabe CS, de Andrade LFC, da Silva MQ No, dos Santos SFT, Kawata LS. Oxigenoterapia domiciliar prolongada: perfil dos usuários e custos. Rev Enferm. 2015;23(1):95-101.

23. Quintão M, Bastos AF, da Silva LM, Bernardez S, Martins W A, Mesquita ET, et al. Ventilação não Invasiva na Insuficiência Cardíaca. Rev da SOCERJ. 2009;22(6):387-97.

24. Bittencourt HS, Reis HF, Lima MS, Gomes M No. Non-invasive ventilation in patients with heart failure: a systematic review and meta-analysis. Arq Bras Cardiol. 2017 Fev;108(2):161-8. PMid:28099587.

25. Soares GMT, Ferreira DCDS, Gonçalves MPC, Alves TG S, David FL, Henriques KM C, et al. Prevalência das principais complicações pós-operatórias em cirurgias cardíacas. Rev Bras Cardiol. 2011;24(3):139-46.

26. Orzechowski R, Galvão AL, Nunes TS, Campos LS. Palliative care need in patients with advanced heart failure hospitalized in a tertiary hospital. Rev Esc Enferm USP. 2019;53:1-6. http://dx.doi.org/10.1590/s1980-220x2018015403413. PMid:30726335.

27. Brant LCC, Nascimento BR, Passos VMA, Duncan BB, Bensenõr IJM, Malta DC, et al. Variações e diferenciais da mortalidade por doença cardiovascular no Brasil e em seus estados, em 1990 e 2015: Estimativas do Estudo Carga Global de Doença. Rev Bras Epidemiol. 2017;20(Suppl 1):11628. http://dx.doi.org/10.1590/1980-5497201700050010. PMid:28658377. 